\section{How to test special relativity}

SIR-In the light of the recent debate in Nature about possible tests of special relativity ${ }^{1-3}$, we wish to direct attention to a relevant question posed in Nature in 1968 by A.C.W.V. Clarket: "What preference is to be given or denied to [the Earth's reference] frame?"

To the best of our knowledge, no subsequent paper in Nature has addressed this simple question. We submit that the physical significance of this neglected problem is of a fundamental character, and we briefly explain below how the quest for the answer points to new important tests of special relativity.

The latter theory was built largely on the basis of the following ideas: Owing to the Earth's known orbital motion, the terrestrial laboratory was considered to be a moving frame of reference. Moreover, owing to the continuous change in direction of the Earth's velocity, it was tacitly assumed that the terrestrial laboratory constitutes different reference frames in different seasons (assumption A).

So when the velocity of light was measured (in the terrestrial laboratory), and its value $c$ was discovered to be paradoxically the same in every direction and in every season, Einstein drew his well known conclusions: first, that $c$ may be referred to any and every reference frame; and second, that all frames are therefore equivalent.

If assumption A is granted, this is sound reasoning. Now in kinematics assumption $\mathrm{A}$ is quite obviously true indeed. However, despite its apparent plausibility, there is considerable evidence ${ }^{5-10}$ casting doubt on its general validity in many aspects of dynamics, electromagnetics, and optics. In these fields there is some cause to resort to assumption $\mathrm{B}$, that the terrestrial laboratory constitutes at all times one and only one reference frame, namely the unique Earth's frame.

When $c$ is measured in the terrestrial laboratory, the terrestrial environment (atmosphere, gravitational and electromagnetic fields and so on) remains practically unaltered in every season, despite the Earth's orbital and other motions. Because of its size, the Earth exerts autonomy over its domain of influence, and there is no gravitational or electromagnetic field wind, nor a wind of any other kind. So in considerations of this kind, assumption $\mathrm{A}$ fails and assumption B seems to be valid.

For these and other reasons, it has been suggested ${ }^{9}$ that the correct interpretation of Maxwell's electromagnetic theory of light might be to postulate that the velocity $c$ of light (Maxwell's electromagnetic waves) has to be referred to the rest frame of the ambient electromagnetic field something like 5 per cent of NK cells: in the rest, although the $\beta$-chain genes are rearranged and transcribed ${ }^{34}$, they give rise only to truncated RNA that cannot participate in the formation of a functional receptor." The data in ref. 34 (Reynolds, C.W. et al., J. exp. Med. 161, 1249; 1985) were misquoted. In fact, the title of the article is "Lack of gene rearrangement and mRNA expression of the $\beta$-chain of the $T$ cell receptor in spontaneous rat large granular lymphocyte leukemia lines." Furthermore, Ritz et al. ${ }^{2}$ have demonstrated that human CD3- NK clones do not rearrange the $\beta$-chain genes. Recently, we isolated CD3-, CD16+ NK cells directly from peripheral blood and have proven that this population does not rearrange the $\beta$-chain genes ${ }^{3}$. Although a truncated $\beta$-chain mRNA may be present in CD3- NK cells, B cell lines also produce abortive $\beta$-chain transcripts ${ }^{4}$.

(2) It is implied that CD3- NK cells are simply stage I thymocytes, based on the observation that NK cells express CD2, but usually lack CD4 and CD8. By extrapolation, should the reader also conclude that monocytes and a subset of $T$ cells are ontogenetically related, since both express the CD4 antigen? There is no experimental evidence indicating that a CD3- peripheral blood NK cell can be induced to rearrange $\mathrm{T}$-cell antigen receptor genes or differentiate into CD3 + T lymphocytes. Although NK cells and T cells may share a common bone marrow precursor cell, there is no evidence to suggest that NK cells arise from the thymus.

(3) "A careful analysis of T-cell receptor gene expression in parallel with physiological studies on NK cells has now confirmed their membership of the T-cell lineage, but seriously damaged their credibility as a legitimate T-cell subset." Since CD3- NK cells do not rearrange Tcell antigen receptor $\beta$-chain genes, and cannot be induced to differentiate into $\mathrm{CD} 3+$ cells, what is the basis for concluding that they have been definitively assigned to the T-cell lineage? Moreover, the small CD3 $+\mathrm{T}$ cell subpopulation that does mediate non-MHC restricted cytotoxicity, productively rearranges and transcribes the T-cell antigen receptor genes, and recognizes antigen via the $\mathrm{CD} 3 / \mathrm{Ti}$ complex ${ }^{3}$. We propose that these nonMHC restricted cytotoxic $\mathrm{T}$ lymphocytes do in fact constitute a legitimate T-cell subset, and should be referred to as "nonMHC restricted CTL", rather than NK cells.

Perhaps the underlying cause for this confusion is that there has been no precise definition for an NK cell. Unfortunately, any lymphoid population killing K562 or YAC has been called an "NK cell" or "NK-like" cell. However, the functional, antigenic, and molecular genetic studies on both freshly isolated lymphocytes and 